\title{
Um Framework para Desenvolvimento de Softwares de Suporte ao Ensino para Plataformas Móveis
}

\author{
Miguel Ângelo Rodrigues Mocbel, UFPA, angelomocbel@gmail.com \\ Vicente Caldas de Castro, UFPA, vicente_soad@yahoo.com.br \\ Laciene Alves Melo, UFPA, lacienealvesmelo@gmail.com \\ Leonardo Nunes Gonçalves, UFPA, leo.widgeon16@gmail.com \\ Marco Aurelio Silva Costa, UFPA, marcosilvacosta54@gmail.com \\ Carlos dos Santos Portela, UFPA, csp@ufpa.br \\ Fabricio de Souza Farias, UFPA, fabriciosf@ufpa.br
}

\begin{abstract}
Resumo. A educação é uma das áreas que vêm aderindo cada vez mais ao uso da tecnologia como suporte à realização de suas tarefas. Uma forma de acesso aos conteúdos educativos é através do uso de dispositivos móveis, como smartphones e tablets. Este tipo de acesso é denominado de aprendizagem com mobilidade ou mobilelearning (m-learning). O presente trabalho apresenta o FRAmework de Suporte ao Ensino (FRASE) voltado ao desenvolvimento de aplicativos m-learning. Para a validação desse framework, implementou-se um aplicativo abordando conteúdos de Ciências Exatas. $O$ aplicativo foi submetido à avaliação via questionário e os resultados obtidos demonstraram altos índices de aceitação por parte dos usuários em relação aos itens de interface de usuário, aprendizagem, organização e satisfação.
\end{abstract}

Palavras-Chave: mobile-learning, aplicativos, framework, ciências exatas.

\section{A Framework for the Development of Teaching Support Software for Mobile Platforms}

\begin{abstract}
Education is one field that is adopting even more the technology to support their tasks. One way to access educational contents can be through mobile technologies, such as smartphones and tablets. This scenario is known as mobile-learning (mlearning). This paper presents a framework for m-learning applications development, called FRASE. In order to assess the work, one application based on FRASE and composed by exact sciences contents were implemented. The application was evaluated through a questionnaire and the obtained results showed high acceptance rates about interface, learning, organization and satisfaction.
\end{abstract}

Keywords: mobile-learning, applications, framework, exact science.

\section{Introdução}

A educação se tornou uma das áreas em que a tecnologia da informação obteve alta aplicabilidade. O computador, a internet e outros recursos eletrônicos se tornaram grandes aliados no processo de ensino-aprendizagem, resultando em diferentes modalidades de ensino como a Educação a Distância (EAD). A adesão da EAD tem crescido a cada ano, como apontam os dados do Censo da Educação Superior de 2018 (INEP, 2018), em que matrículas na modalidade já alcançam $24,3 \%$ do total de matrículas no ensino superior, atingindo mais de 2 milhões de estudantes. Uma forma de ensino fundamentada no uso da tecnologia para acesso aos conteúdos de EAD é a aprendizagem com mobilidade ou Mobile-Learning (m-learning) (Georgiev, Georgieva e Smrikarov, 2004). 
Com o avanço das tecnologias móveis, o m-learning estendeu o conceito do aprendizado eletrônico, pois além de permitir o acesso às plataformas de $\mathrm{EAD}$, possui um conceito mais amplo, no qual é possível disponibilizar o acesso ao conhecimento de forma independente a qualquer instituição. Esta liberdade possibilita ao usuário acessar conteúdos, exercícios e vídeos provenientes de diferentes fontes, a partir de vários aplicativos desenvolvidos para plataformas móveis que abordam sobre determinado assunto. Assim, a característica principal do m-learning constitui-se no livre acesso ao conhecimento diverso em qualquer momento e em qualquer lugar (Georgiev, Georgieva e Smrikarov, 2004) (Franciscato e Medina, 2008).

$\mathrm{Na}$ literatura, diversos trabalhos propõem novas ferramentas que visam apoiar o processo de aprendizado a partir de plataformas móveis. Há trabalhos que envolvem o conceito de m-learning e apresentam protótipos de aplicações educacionais para atender diferentes disciplinas de estudo (Castro et al., 2016) (Machado et al., 2018). Além disso, autores como Silva et al. (2015) afirmam que incluir ferramentas baseadas em tecnologias da informação em sala de aula pode resultar no desenvolvimento de várias habilidades dos alunos e, que dependendo do modo que o professor faz proveito dessas ferramentas, pode possibilitar a melhoria do processo educativo.

No trabalho de Castro et al. (2016), a proposta de aplicação m-learning foi voltada para o contexto do aluno. A pesquisa apresentou o desenvolvimento de um sistema de aprendizagem para plataformas móveis abordando assuntos de matemática e informática para o ensino médio. Nesta vertente, Machado et al. (2018) propuseram um aplicativo móvel para auxiliar o aprendizado de programação baseado no conceito colaborativo. A partir da análise dos resultados da pesquisa, foi possível concluir que o aplicativo proposto é capaz de reduzir o índice de reprovações, assim como, pode ser considerado um fator estimulante, garantindo a aprendizagem via colaboração entre os alunos.

Além das pesquisas para o desenvolvimento e teste de novos softwares, diversos trabalhos têm investigado os benefícios da utilização de plataformas móveis como ferramenta de suporte ao processo de ensino-aprendizagem no ensino médio, por exemplo, Rodriguez et al. (2016), Batista e Barcelos (2017) e Nichele e Schlemmer (2014). A investigação neste contexto se faz necessária, pois de acordo com Oliveira e Medina (2007), o uso de ferramentas móveis no ensino pode proporcionar mais interação e dinamismo entre os agentes envolvidos.

Em relação à avaliação de soluções de softwares para o ensino médio, Rodriguez et al. (2016) realizaram uma análise técnica e ressaltaram a importância da usabilidade em ferramentas de m-learning. Em paralelo, Batista e Barcelos (2017) declaram que o smartphone tem trazido mais praticidade para o cotidiano em diversos momentos e que o uso adequado do dispositivo pode trazer contribuições para o âmbito educacional. Na área da educação, Nichele e Schlemmer (2014) e Perez, Viali e Lahm (2015) apresentam trabalhos que analisam diferentes conteúdos disponibilizados em diversas ferramentas. $\mathrm{O}$ primeiro avalia o cenário de aplicativos voltados para os conteúdos de Química, enquanto que o segundo analisa as aplicações disponíveis para o ensino de Física. Ambos os trabalhos expõem as potencialidades que ferramentas móveis podem ter no ensino, transformando metodologias quando aliadas às práticas pedagógicas.

Embora os outros trabalhos apresentem soluções baseadas em m-learning que visam auxiliar os agentes envolvidos no processo de ensino-aprendizagem, muitas propostas são voltadas para uma área de conhecimento específica, por exemplo, aplicações que envolvem somente Matemática (Arantes e Seabra, 2016), somente Física (Perez, Viali e Lahm, 2015) ou somente Química (Nichele e Schlemmer, 2014) (Rocha 
et al., 2017). Sendo assim, esses trabalhos não abordam um aspecto genérico em relação ao conteúdo, e não levam em consideração uma organização entre a composição de telas e funcionalidades.

Diante deste contexto, este artigo tem como objetivo apresentar o FRAmework de Suporte ao Ensino (FRASE). O FRASE visa auxiliar o desenvolvimento de aplicações $m$ learning, sendo utilizado para a implementação de um aplicativo voltado às disciplinas de Ciências Exatas do nível médio, denominado Exatas (Mocbel e Farias, 2018). Para avaliação do Exatas, o mesmo foi publicado na plataforma de aplicativos Google Play, sendo sujeito às avaliações de aceitabilidade com seus usuários, considerando os quesitos de interface de usuário, aprendizagem, organização e satisfação, baseando-se em critérios aderentes às características propostas na norma ISO/IEC 25000 (2014).

O restante deste artigo está organizado em 5 seções. Na Seção 2 são apresentados os aplicativos investigados e o levantamento dos requisitos. Na Seção 3 é apresentada a estrutura de funcionamento do framework. São expostos na Seção 4, o aplicativo desenvolvido a partir do FRASE, o painel para cadastro de conteúdo, bem como a avaliação do aplicativo. Os resultados obtidos a partir da avaliação do aplicativo são discutidos na Seção 5. Por fim, na última seção são apresentadas as conclusões do trabalho e as próximas etapas da pesquisa.

\section{Análise do Projeto}

Durante a etapa de análise foram realizadas pesquisas com o intuito de obter informações de aplicativos educacionais relacionados a proposta do trabalho. Com isso, definiu-se os requisitos para o desenvolvimento do FRASE.

\subsection{Aplicativos Relacionados}

Para complementar a pesquisa, aplicativos de cunho educacional foram instalados e analisados com a finalidade de estudar suas principais características, sendo que os critérios para a busca envolveram gratuidade, popularidade e temática voltada ao Exame Nacional do Ensino Médio (ENEM). A Tabela 1 apresenta os aplicativos destacando sua descrição e tipo de conteúdo.

Tabela 1 - Aplicativos educativos analisados.

\begin{tabular}{|c|c|c|c|c|c|}
\hline \multirow{2}{*}{ APP } & \multirow{2}{*}{ Aplicativo } & \multirow{2}{*}{ Descrição } & \multicolumn{3}{|c|}{ Tipo de conteúdo } \\
\hline & & & Apostilas & Questões & Vídeos \\
\hline APP1 & $\begin{array}{l}\text { Studos - Enem } \\
\text { e Vestibulares }\end{array}$ & Simulados baseados no ENEM. & $\mathrm{X}$ & & \\
\hline APP2 & Me Salva! & Plataforma de cursos preparatórios. & & $\mathrm{X}$ & $\mathrm{X}$ \\
\hline APP3 & G1 ENEM & $\begin{array}{l}\text { Listas de exercícios baseados no ENEM } \\
\text { e vídeos relacionados. }\end{array}$ & & & $\mathrm{X}$ \\
\hline APP4 & Brainly & $\begin{array}{l}\text { Formato de perguntas e respostas nas } \\
\text { mais diversas áreas do conhecimento. }\end{array}$ & & & $\mathrm{X}$ \\
\hline APP5 & História & Concentra material de fatos históricos. & $\mathrm{X}$ & & \\
\hline APP6 & Udemy & Plataforma de cursos. & & & $\mathrm{X}$ \\
\hline APP7 & Descomplica & Plataforma de cursos preparatórios. & $\mathrm{X}$ & & $\mathrm{X}$ \\
\hline APP8 & $\begin{array}{l}\text { Nota } 10 ! \\
\text { Simulados do } \\
\text { Enem }\end{array}$ & $\begin{array}{l}\text { Listas de exercícios baseados no } \\
\text { ENEM. }\end{array}$ & $\mathrm{X}$ & & \\
\hline
\end{tabular}


É possível observar que, entre os aplicativos analisados, nenhum disponibiliza os três tipos de conteúdo, e a maioria das aplicações são baseadas em vídeos e poucas dispõem de material textual, o que revela uma lacuna a ser explorada.

\subsection{Levantamento de Requisitos}

Os requisitos para o FRASE foram definidos a partir da análise das principais características dos aplicativos educativos dispostos na Tabela 1. Nesta etapa, buscou-se priorizar as funcionalidades mais relevantes, assim como as que não são exploradas. $\mathrm{Na}$ Tabela 2, encontram-se os principais requisitos organizados em código (R\#), funcionalidade, descrição e aplicativos referentes ao requisito.

Tabela 2 - Principais requisitos funcionais do FRASE.

\begin{tabular}{|c|c|c|c|}
\hline $\mathbf{R \#}$ & Funcionalidade & Descrição & Aplicativos \\
\hline R01 & $\begin{array}{l}\text { Autenticação de } \\
\text { usuário }\end{array}$ & $\begin{array}{l}\text { O aplicativo deve permitir ao usuário se registrar e } \\
\text { entrar com suas credenciais. }\end{array}$ & $\begin{array}{l}\text { APP1, APP2, APP3, } \\
\text { APP4, APP6, APP7 e } \\
\text { APP8 }\end{array}$ \\
\hline R02 & $\begin{array}{l}\text { Listagem de } \\
\text { itens }\end{array}$ & O aplicativo deve permitir ao usuário acessar itens. & APP2 e APP3 \\
\hline $\mathrm{R} 03$ & $\begin{array}{l}\text { Listagem de } \\
\text { subitens }\end{array}$ & $\begin{array}{l}\text { O aplicativo deve permitir ao usuário acessar e } \\
\text { buscar subitens. }\end{array}$ & APP2 \\
\hline R04 & $\begin{array}{l}\text { Consulta de } \\
\text { material textual }\end{array}$ & $\begin{array}{l}\text { O aplicativo deve permitir ao usuário acessar o } \\
\text { material textual de um subitem. }\end{array}$ & APP5 e APP7 \\
\hline R05 & $\begin{array}{l}\text { Resolução de } \\
\text { questões }\end{array}$ & $\begin{array}{l}\text { O aplicativo deve permitir ao usuário responder } \\
\text { listas de questões relacionadas a um subitem. }\end{array}$ & $\begin{array}{c}\text { APP1, APP3, APP4 e } \\
\text { APP8 }\end{array}$ \\
\hline R06 & $\begin{array}{l}\text { Consulta de } \\
\text { videoaulas }\end{array}$ & $\begin{array}{l}\text { O aplicativo deve permitir ao usuário acessar } \\
\text { vídeos relacionados a subitens. }\end{array}$ & $\begin{array}{c}\text { APP2, APP3, APP6 e } \\
\text { APP7 }\end{array}$ \\
\hline R07 & $\begin{array}{l}\text { Consulta de } \\
\text { vídeo-resolução }\end{array}$ & $\begin{array}{l}\text { O aplicativo deve permitir ao usuário acessar } \\
\text { vídeos relacionados a questões. }\end{array}$ & Nenhum \\
\hline R08 & $\begin{array}{l}\text { Consulta de } \\
\text { resultado }\end{array}$ & $\begin{array}{l}\text { O aplicativo deve permitir ao usuário acessar seu } \\
\text { quantitativo de acertos e erros em uma lista de } \\
\text { questão. }\end{array}$ & APP1, APP3 e APP8 \\
\hline R09 & $\begin{array}{l}\text { Consulta de } \\
\text { desempenho } \\
\text { geral }\end{array}$ & $\begin{array}{l}\text { O aplicativo deve permitir ao usuário acessar o seu } \\
\text { desempenho percentual relacionado as questões } \\
\text { resolvidas. }\end{array}$ & APP1, APP3 e APP8 \\
\hline $\mathrm{R} 10$ & $\begin{array}{l}\text { Sincronização } \\
\text { de desempenho }\end{array}$ & $\begin{array}{l}\text { O aplicativo deve permitir ao usuário salvar seu } \\
\text { desempenho em nuvem, bem como realizar tal } \\
\text { tarefa de modo automático. }\end{array}$ & APP1 e APP3 \\
\hline
\end{tabular}

Com base na Tabela 2, é possível perceber que nenhum aplicativo esteve referenciado em todos os requisitos definidos para o framework proposto. Destaca-se também o R07 sobre vídeo-resolução por não possuir nenhuma citação entre os softwares analisados. Assim, percebe-se a abrangência funcional da proposta com base no cenário de aplicativos educativos.

\section{FRAmework de Suporte ao Ensino (FRASE)}

Entende-se como um framework uma ferramenta capaz de agrupar um conjunto de funcionalidades genéricas que visam suprir requisitos comuns a diferentes contextos e com códigos-fonte reutilizáveis. O FRASE consiste em um conjunto de requisitos e estruturas que definem como uma aplicação m-learning pode ser implementada. $\mathrm{O}$ 
objetivo do FRASE é permitir ao desenvolvedor a construção de aplicações educacionais compostas por conteúdos que podem ser adaptados para a área de conhecimento abordada, e que os produtos implementados a partir desse framework possam ser utilizados em sala de aula, em ambientes extraclasse acompanhado por um tutor, ou para uso individual (seja pelo professor ou aluno).

\subsection{Estrutura Geral do FRASE}

O FRASE é uma plataforma que visa reunir diferentes funcionalidades contidas em soluções baseadas em m-learning para suporte a conteúdos de diferentes áreas de estudo. Este traz como diferencial, entre os aplicativos analisados, uma organização que dispõe os conteúdos de forma híbrida, ou seja, conteúdo em texto disponível de forma offline, e questões e vídeos disponíveis de forma online, o que reduz o tamanho (espaço ocupado na memória) de qualquer aplicação criada pelo framework, assim como remove a necessidade de atualização do software em caso de disponibilidade de novas questões e vídeos. A Figura 1 apresenta a estrutura geral do FRASE.

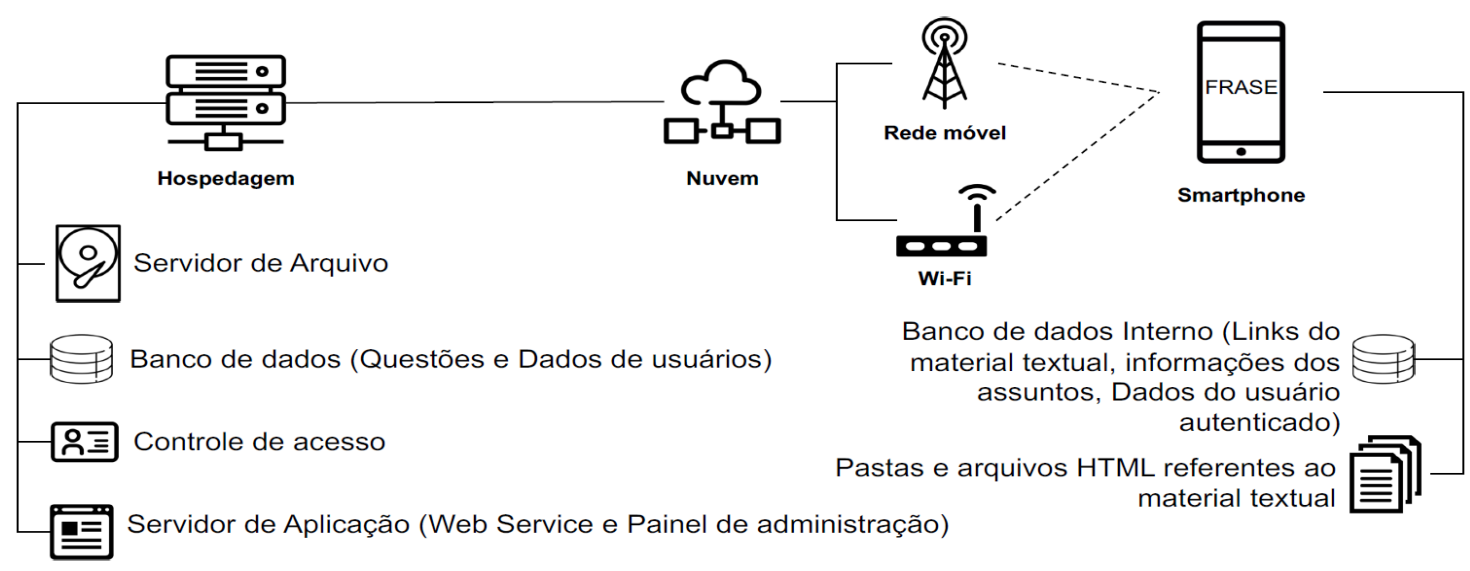

Figura 1 - Estrutura geral do FRASE.

Conforme a Figura 1, do lado do usuário existe um banco de dados interno, que armazena as informações de itens e indexação do material textual para disponibilização offline no aplicativo. No lado dos servidores é encontrado o servidor de controle de acesso (responsável em persistir as informações do usuário e do servidor), servidor de aplicação (responsável em armazenar o website que recebe os conteúdos administrados/adicionados pelo gerenciador da aplicação) e servidor de banco de dados (responsável em armazenar os dados dos usuários, questões cadastradas e links de vídeos).

A implementação do FRASE se deu na linguagem de programação Java, através do ambiente de desenvolvimento integrado (IDE) Android Studio. Os servidores utilizados pelo framework foram hospedados no serviço de computação em nuvem da Amazon. As aplicações instaladas nos servidores foram programadas na linguagem de programação PHP e o sistema gerenciador de banco de dados utilizado foi o MySQL.

\section{Caso de Estudo}

Para a demonstração da aplicabilidade do framework desenvolvido, implementouse um aplicativo reutilizando o código-fonte do FRASE. O software foi denominado Exatas, sendo o primeiro que incorporou os requisitos e estrutura geral do FRASE, isto é, implementando os requisitos de R01 ao R10. O nome Exatas faz referência a área de 
conhecimento escolhida, mais especificamente, assuntos de nível médio das disciplinas Matemática, Física e Química. O Exatas é organizado a partir de um menu de opções posicionado na lateral esquerda da tela, vide Figura 2 (a). Na tela inicial, conforme Figura 2 (b), são apresentadas as disciplinas, que ao serem clicadas, retornam ao usuário a lista de assuntos disponíveis para uma disciplina, como pode ser visto na Figura 2 (c).

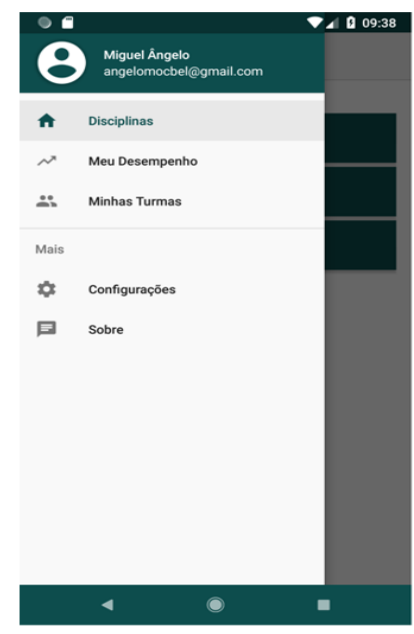

(a)

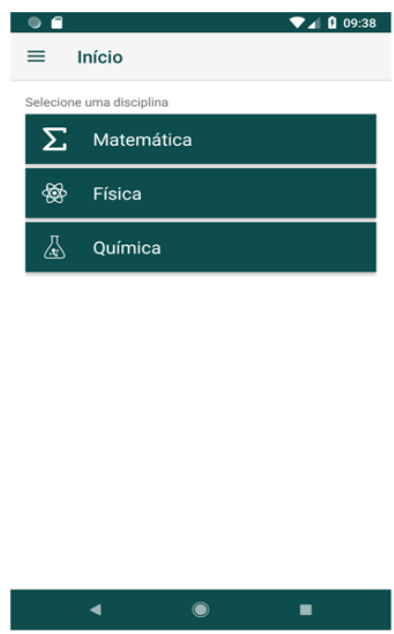

(b)

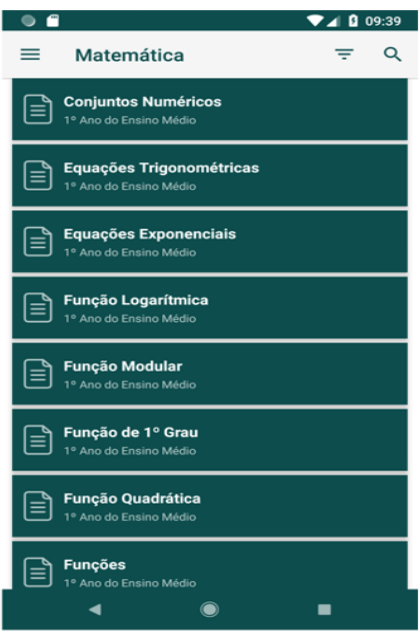

(c)

Figura 2 - Telas do Exatas. (a) Menu Lateral. (b) Tela Inicial. (c) Lista de Assuntos.

Ao selecionar um item na lista de assuntos (Figura 2 (c)), o usuário é direcionado para uma tela, onde são apresentadas as seguintes abas: Conteúdo, Questões e Vídeos. Na primeira aba, conforme Figura 3 (a), que já é carregada assim que a tela é aberta, o aluno tem acesso ao conteúdo textual do assunto selecionado, onde é permitido navegar e aplicar zoom sobre o texto em exibição. Ao clicar na aba Questões, o aluno é direcionado para uma tela que contém duas opções: a primeira diz respeito ao início da apresentação da lista de exercícios; a segunda opção permite ao usuário configurar o número de itens de questões que serão apresentados nas listas de exercícios. Por fim, a aba Vídeos, apresenta ao usuário uma lista de opções de vídeos que possuem informações teóricas sobre o assunto em estudo.

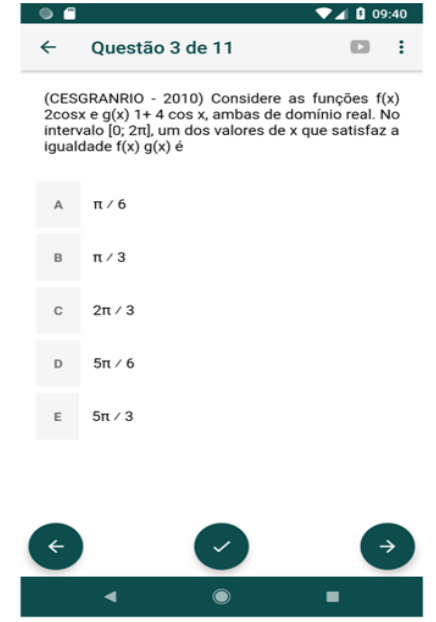

(a)

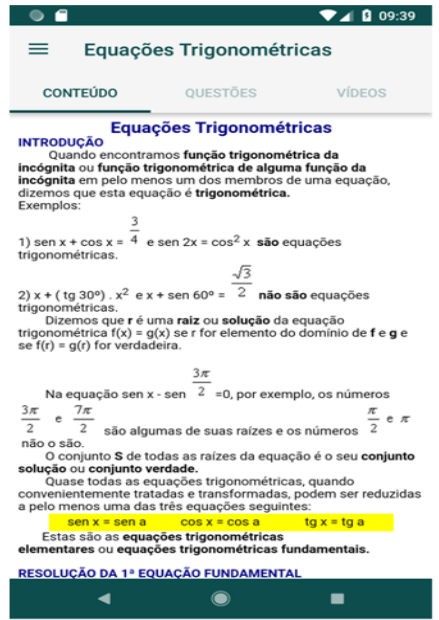

(b)

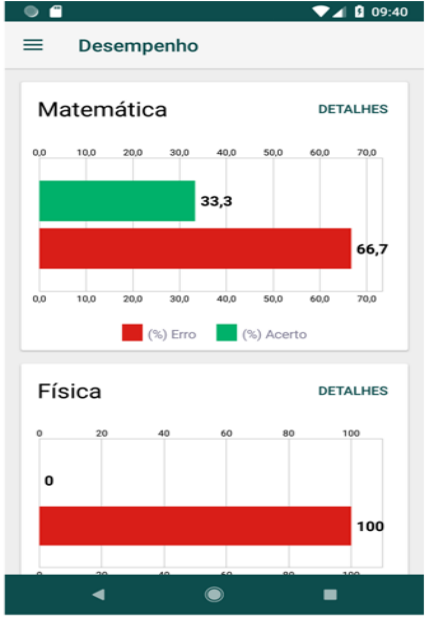

(c)

Figura 3 - Principais telas do Exatas. (a) Abas Conteúdo, Questões e Vídeos. (b) Lista de questões iniciada. (c) Desempenho do Usuário. 
A Figura 3 (b) ilustra um exemplo de lista de exercícios iniciada por um usuário. Questões aleatórias relacionadas ao assunto escolhido na tela anterior são carregadas de um banco de dados na internet conforme o número de questões configurado na aba Questões. As questões são exibidas uma a uma na tela, estando acessíveis através de dois botões flutuantes na parte inferior da tela com ícones de seta, permitindo a navegação entre as questões. Para resolver uma questão, é necessário clicar sobre uma opção, selecionando-a, para então submeter a resposta no botão inferior que se encontra centralizado aos botões de navegação de questão. Dessa forma, é possível consultar o desempenho contabilizado a partir da resolução de listas de exercícios, que é exibido em gráficos distribuídos por disciplina, conforme Figura 3 (c). Os gráficos apresentam os índices de acertos e erros na forma de porcentagem. Ao clicar no botão Detalhes de um gráfico, são apresentados os índices absolutos de erros e acertos, e um detalhamento gráfico de desempenho referente a cada ano escolar.

Para avaliação do aplicativo desenvolvido a partir do FRASE, foi elaborado um formulário que envolveu questões sobre interface de usuário, aprendizagem, organização e satisfação. Optou-se por dispor tais questões em formato de afirmações, no qual o usuário pode informar o grau de concordância com as mesmas. Esse formato de formulário tem como base a escala de Likert (1932), e os itens de avaliação adotados foram baseados nas características de usabilidade da ISO/IEC 25000 (2014). O formulário foi disponibilizado durante quatro meses para os usuários do aplicativo Exatas, nos quais aqueles que fizeram uso de duas ou mais funcionalidades da aplicação foram convidados a responder a pesquisa, assim, coletando respostas de usuários ativos do software. Para cada afirmação do questionário, o usuário pôde avaliar atribuindo um nível de concordância entre 5 opções: (1) Discordo totalmente; (2) Discordo parcialmente; (3) Indiferente; (4) Concordo parcialmente; e (5) concordo totalmente.

\section{Resultados}

Esta seção apresenta os resultados obtidos a partir do software criado com o FRASE, sendo ele, o Exatas. Os dados foram coletados a partir do formulário disponibilizado dentro do aplicativo, visando avaliar a aceitação da ferramenta desenvolvida. Os dados coletados a partir do formulário aplicado ao Exatas totalizaram 4156 respostas de usuários considerados ativos da aplicação. As respostas foram contabilizadas e dispostas em tabelas organizadas em número da afirmação (A\#), item de avaliação e níveis de concordância.

A Tabela 3 apresenta os resultados do quesito Interface de Usuário, Aprendizagem, Organização e Satisfação. Para a análise da Interface do Usuário, foi considerada a forma de apresentação do conteúdo (A1), capacidade de manutenção da atenção do usuário (A2) e disposição e adequação das informações na tela (A3). A partir dos resultados, é possível identificar percentuais positivos de concordância e concordância total para as afirmativas A1, A2 e A3, obtendo os respectivos índices de concordância, 85\%, 87\% e 89\%, para o software Exatas. Os resultados indicam que a identidade visual proposta no FRASE se adequa a diferentes níveis de ensino e públicos.

Sobre o quesito Aprendizagem, foi levado em consideração a resposta da aplicação como suporte a aprendizagem (A4), contribuição para aprendizagem de conhecimento e de novas habilidades (A5), capacidade de autonomia de aprendizagem na plataforma (A6), e facilitação do processo de aprendizagem (A7). A partir dos resultados, é possível identificar um percentual médio de concordância e concordância total acima de $85 \%$. Esses resultados refletem estudos como a pesquisa de Silva (2011), 
que considera as tecnologias como ferramentas aptas a ampliar a capacidade cognitiva dos indivíduos por meio da disponibilização do acesso às informações em qualquer momento e em qualquer lugar. Nesse sentido, é importante destacar o potencial do FRASE enquanto ferramenta de produção de softwares para suporte ao ensino, como o Exatas.

Tabela 3 - Avaliação sobre Interface do usuário, aprendizagem, organização e satisfação.

\begin{tabular}{|c|c|c|c|c|c|c|}
\hline A\# & Itens de avaliação do aplicativo Exatas & 1 & 2 & 3 & 4 & 5 \\
\hline A1 & $\begin{array}{l}\text { A forma da apresentação dos textos e } \\
\text { imagens me incentiva a utilizar o aplicativo. }\end{array}$ & $2,65 \%$ & $3,37 \%$ & $8,33 \%$ & $34,79 \%$ & $50,87 \%$ \\
\hline A2 & $\begin{array}{l}\text { A interface gráfica do aplicativo é atraente e } \\
\text { mantém sua atenção. }\end{array}$ & $1,97 \%$ & $2,33 \%$ & $8,54 \%$ & $30,75 \%$ & $56,40 \%$ \\
\hline A3 & $\begin{array}{l}\text { A quantidade de informações apresentadas } \\
\text { em cada tela do aplicativo é adequada e } \\
\text { suficiente para um bom aprendizado. }\end{array}$ & $1,64 \%$ & $1,88 \%$ & $7,12 \%$ & $24,11 \%$ & $65,26 \%$ \\
\hline A4 & $\begin{array}{l}\text { O feedback de desempenho oferecido após as } \\
\text { resoluções dos exercícios dispostos no } \\
\text { aplicativo auxilia no entendimento das } \\
\text { atividades e na melhoria do aprendizado. }\end{array}$ & $1,73 \%$ & $3,20 \%$ & $10,44 \%$ & $31,18 \%$ & $53,44 \%$ \\
\hline A5 & $\begin{array}{l}\text { O aplicativo fornece contribuição útil para a } \\
\text { construção de conhecimentos e habilidades } \\
\text { de forma independente ao ambiente de sala de } \\
\text { aula. }\end{array}$ & $1,42 \%$ & $1,20 \%$ & $5,85 \%$ & $21,94 \%$ & $69,59 \%$ \\
\hline A6 & $\begin{array}{l}\text { A teoria repassada em sala de aula sobre o } \\
\text { conteúdo abordado pode ser reforçada no } \\
\text { aplicativo. }\end{array}$ & $1,85 \%$ & $2,26 \%$ & $7,72 \%$ & $28,15 \%$ & $60,01 \%$ \\
\hline A7 & $\begin{array}{l}\text { O aplicativo facilita a fixação de } \\
\text { conhecimentos e habilidades abordadas em } \\
\text { sala de aula. }\end{array}$ & $3,27 \%$ & $6,47 \%$ & $12,13 \%$ & $29,76 \%$ & $48,36 \%$ \\
\hline A8 & $\begin{array}{l}\text { As organizações metodológicas das } \\
\text { informações apresentadas no aplicativo } \\
\text { aumentam a compreensão dos conteúdos. }\end{array}$ & $1,59 \%$ & $2,02 \%$ & $11,19 \%$ & $28,06 \%$ & $57,15 \%$ \\
\hline A9 & $\begin{array}{l}\text { O conteúdo abordado é bem organizado e } \\
\text { fácil de entender no aplicativo. }\end{array}$ & $2,96 \%$ & $4,86 \%$ & $11,07 \%$ & $26,18 \%$ & $54,93 \%$ \\
\hline A10 & $\begin{array}{l}\text { Qual seu nível de satisfação com o } \\
\text { aplicativo. }\end{array}$ & $2,77 \%$ & $4,67 \%$ & $11,65 \%$ & $26,64 \%$ & $54,28 \%$ \\
\hline A11 & Você indicaria o aplicativo para um amigo. & $1,32 \%$ & $0,82 \%$ & $4,14 \%$ & $17,44 \%$ & $76,28 \%$ \\
\hline $\mathrm{A} 12$ & Usaria o aplicativo. & $2,09 \%$ & $1,30 \%$ & $5,87 \%$ & $17,08 \%$ & $73,65 \%$ \\
\hline A13 & $\begin{array}{l}\text { O aplicativo é apropriado para os estudos do } \\
\text { conteúdo abordado. }\end{array}$ & $1,47 \%$ & $1,42 \%$ & $9,41 \%$ & $29,72 \%$ & $57,99 \%$ \\
\hline
\end{tabular}

Referente ao quesito Organização, considerou-se a capacidade de compreensão a partir da organização das informações (A8) e a facilitação de entendimento a partir do modo de organização dos conteúdos (A9). Os resultados apontam índices de concordância e concordância total acima de $81 \%$. Por fim, sobre o quesito Satisfação, foram analisadas questões como: indicação da ferramenta para outros usuários (A11), adoção do software (A12) e apropriação da aplicação como ferramenta de estudo (A13). Seguindo estes critérios, o aplicativo Exatas obteve índices de concordância e concordância total para as afirmações A10, A11, A12 e A13 de, respectivamente, 83\%, 93\%, 90\% e 87\%. Este fato reforça o trabalho de Oliveira et al. (2017), o qual destaca que a tecnologia enquanto 
instrumento pedagógico funciona de forma transformadora nos ambientes que a adotam. Em suma, a partir dos resultados obtidos, é possível verificar que o uso do FRASE permite a criação de ferramentas com potencial de aceitação entre seus usuários, o que possibilita futuras investigações sobre o uso dessas tecnologias no processo de ensinoaprendizagem, seja para o uso individual do aluno, ou em sala de aula mediado por um professor.

\section{Conclusões e Trabalhos Futuros}

Este artigo apresentou o FRASE, acrônimo para Framework de Suporte ao Ensino. O FRASE é voltado à criação de softwares para suporte ao ensino baseado em $m$ learning. A partir do levantamento de aplicativos realizado neste trabalho, vide Tabela 1, pode-se concluir que o FRASE possui as principais funcionalidades já apresentadas de forma dispersa em diferentes aplicativos, assim como, é um framework de suporte ao ensino. Para validar o framework, um aplicativo foi implementado levando em consideração as funcionalidades do FRASE e, além disso, um formulário de avaliação foi aplicado aos usuários ativos do software. A partir da análise dos resultados obtidos, podese concluir que o FRASE é capaz de conduzir a produção de ferramentas que podem ser aplicadas em processos de ensino-aprendizagem, pois foi estruturado considerando a produção de interfaces gráficas amigáveis e de fácil uso, permitindo a criação de um ambiente de aprendizagem diversificado que pode estimular aspectos cognitivos a partir do uso de conteúdos, questões e vídeos.

Após a avaliação realizada por 4156 usuários, os resultados demonstram que o software desenvolvido a partir do FRASE obteve altos índices de aceitação. No que diz respeito à interface de usuário, o índice médio geral de concordância (valor aproximado da soma dos índices de concordância e concordância total) foi de $87 \%$. Em relação ao quesito aprendizagem, o índice médio geral de concordância foi de $85 \%$. Já para o quesito sobre organização, foi alcançado um índice de $83 \%$. Por fim, o índice de satisfação geral alcançado foi de $89 \%$. A partir dos índices obtidos, é possível afirmar que aplicativos construídos com o FRASE podem vir a ajudar os estudos de alunos e professores do ensino médio ou do ensino superior que usam a tecnologia em seu dia a dia.

Com este trabalho, pode-se concluir que ferramentas baseadas em m-learning são alternativas viáveis para suporte ao aprendizado, como também podem reforçar conhecimentos adquiridos em sala de aula, pois tais ferramentas permitem o acesso, a qualquer momento e em qualquer lugar, a conteúdos de diversos formatos, assim como, possuem baixo ou nenhum custo de aquisição por parte dos usuários. Como trabalhos futuros, espera-se utilizar o framework para atender outras áreas do conhecimento. Além disso, expandir suas funcionalidades com gerenciamento de turmas e aplicação de simulados.

\section{Referências}

ARANTES, H.; SEABRA, R. D. Aplicativo M-Learning para o Estudo de Conceitos Matemáticos para o ENEM. Em Revista de Sistemas e Computação (RSC), v. 7, n. 1, 2017.

BATISTA, S.; BARCELOS, G. Análise do Uso do Celular no Contexto Educacional. Em Revista Novas Tecnologias na Educação (RENOTE), v. 11, n. 1, 2017.

CASTRO, G.; DOMINGUEZ, E.; VELAZQUEZ, Y.; MATLA, M.; TOLEDO, C.; HERNANDEZ, S. Mobilearn: Context-aware Mobile Learning System. IEEE Latin America Transactions, v. 14, n. 2, p. 958-964, 2016. 
FRANCISCATO, F.; MEDINA, R. M-Learning e Android: Um Novo Paradigma? . Em Revista Novas Tecnologias na Educação (RENOTE), v. 6, n. 1, 2008.

GEORGIEV, T.; GEORGIEVA, E.; SMRIKAROV, A. M-learning-a New Stage of ELearning. In: International Conference on Computer Systems and Technologies (CompSysTech), v. 4, n. 28, p. 1-4, 2004.

INEP. Notas sobre o Censo da Educação Superior. 2018. Disponível em: $<$ http://download.inep.gov.br/educacao_superior/censo_superior/documentos/2019/cens o_da_educacao_superior_2018-notas_estatisticas.pdf>. Acesso em: 15 mar. 2020.

ISO/IEC. ISO/IEC 25000: Systems and Software Engineering - Systems and software Quality Requirements and Evaluation (SQuaRE). 2014. Disponível em: < https://www.iso.org/standard/64764.html>. Acesso em: 15 mar. 2020.

LIKERT, R. A Technique for the Measurement of Attitudes. Archives of Psychology, 1932.

MACHADO, L.; BERKENBROCK, C.; ANSELMO, G.; SIPLE, I. Uma Ferramenta Colaborativa para Apoiar a Aprendizagem de Programação de Computadores. Em Revista Brasileira de Computação Aplicada (RBCA), v. 10, n. 1, p. 23-29, 2018.

MOCBEL, M.; FARIAS, F. Aplicativo Exatas: Ferramenta de apoio ao aprendizado móvel. In: Anais dos Workshops do VII Congresso Brasileiro de Informática na Educação (WCBIE), p. 172-179, 2018.

NICHELE, A.; SCHLEMMER, E. Aplicativos para o ensino e aprendizagem de Química. Em Revista Novas Tecnologias na Educação (RENOTE), v. 12, n. 2, 2014.

OLIVEIRA, G.; KUNRATH, N.; ROCHA, S.; DA SILVA, L. Uso de Dispositivos Móveis como Recurso Didático na Formação Docente. In: WORKSHOP DE INFORMÁTICA NA ESCOLA (WIE), Recife, 2017, p. 725-734.

OLIVEIRA, L.; MEDINA, R. Desenvolvimento de Objetos de Aprendizagem para Dispositivos Móveis: uma Nova Abordagem que Contribui para a Educação. Em Revista Novas Tecnologias na Educação (RENOTE), v. 5, n. 1, 2007.

PEREZ, M.; VIALI, L.; LAHM, R. Aplicativos para Tablets e Smartphones no Ensino de Física. Revista Ciências \& Ideias, v. 7, n. 1, p. 154-173, 2015.

ROCHA, F.; ROCHA, D.; MONÇÃO, N.; NETO, R.; COSTA, J.; FARIAS, K.; LIMA, B.; SANTANA, A. AbaQuim-Um Jogo Educativo para Auxílio na Aprendizagem de Distribuição Eletrônica Química. In: SIMPÓSIO BRASILEIRO DE INFORMÁTICA NA EDUCAÇÃO (SBIE), Recife, 2017, p. 907-916.

RODRIGUEZ, A.; DOMINGUEZ, E.; VELAZQUEZ, Y.; NIETO, M. Usability Assessment of Mobile Learning Objects by High School Students. IEEE Latin America Transactions, v. 14, n. 2, p. 1044-1049, 2016.

SILVA, F.; DE SOUZA, A.; DOS SANTOS, A.; DOS SANTOS, B.; FERREIRA, M. Aplicação e Avaliação do Software Educacional Labvirt: Estudo de Caso numa Turma de Graduação em Química. In: CONGRESSO DE INOVAÇÃO PEDAGÓGICA EM ARAPIRACA, Arapiraca, 2015, p. 1-13.

SILVA, L. A Utilização dos Recursos Tecnológicos no Ensino Superior. Olhar Científico, v. 1, n. 2, p. 267-285, 2011. 